\title{
Consequences of lattice imperfections and interchain couplings for the critical properties of spin- $1 / 2$ chain compounds
}

\author{
J. Sirker \\ ${ }^{1}$ Department of Physics and Research Center OPTIMAS, University of Kaiserslautern, \\ D-67663 Kaiserslautern, Germany \\ 2 Max-Planck Institute for Solid State Research, Heisenbergstr. 1, D-70569 Stuttgart, Germany
}

Received May 7, 2009

To allow for a comparison of theoretical predictions for spin chains with experimental data, it is often important to take impurity effects as well as interchain couplings into account. Here we present the field theory for finite spin chains at finite temperature and calculate experimentally measurable quantities like susceptibilities and nuclear magnetic resonance spectra. For the interchain couplings we concentrate on geometries relevant for cuprate spin chains like $\mathrm{Sr}_{2} \mathrm{CuO}_{3}$ and $\mathrm{SrCuO}_{2}$. The field theoretical results are compared to experimental as well as numerical data obtained by the density matrix renormalization group.

Key words: spin chains, impurities, thermodynamics, bosonization, density-matrix renormalization group

PACS: $75.10 . P q, 75.10 . J m, 11.10 . \mathrm{Wx}, 02.30 . \mathrm{k}$

\section{Introduction}

A large number of materials are known which, over a certain temperature range, are well described by simple spin chain or ladder models [1-11]. In all these Mott insulators the superexchange constants are spatially very anisotropic so that a three-dimensional crystal effectively shows onedimensional magnetic properties. In one of the best known spin- $1 / 2$ chain compounds $\mathrm{Sr}_{2} \mathrm{CuO}_{3}$, for example, the superexchange constant along the chain direction $J \sim 2200 \mathrm{~K}$ whereas the magnetic couplings $J_{\perp}$ along the other directions are at least three orders of magnitude smaller [1,8]. In such a system one can, therefore, experimentally explore the physical properties of a spin chain over a very wide temperature range $J_{\perp} \ll T \lesssim J$. From a theoretical perspective this is very exciting because it allows us to study experimentally many aspects of one-dimensional field theories [12-14]. In addition, the ideal Heisenberg spin- $1 / 2$ chain is integrable so that the compounds well described by this model make it possible to experimentally address the question how physical properties, in particular, transport, are affected by a nearby integrable point [2-4]. Here the infinite set of constants of motion making the model integrable is expected to slow down the decay of current correlations - or even prevent them from decaying completely - leading to anomalous transport properties [15].

In real materials, however, we are always confronted with impurities and lattice imperfections which weaken or even completely destroy a superexchange bond between two spins. Since a weakening of a bond is a relevant perturbation in the renormalization group (RG) sense, we have to deal - at least at low temperatures - with finite chains with open boundary conditions (OBCs). Measurements on such systems correspond to taking averages over ensembles of finite chain segments with lengths determined by a distribution function $[16,17]$. Furthermore, the description by a one dimensional model will break down for temperatures $T \sim J_{\perp}$ where usually a three-dimensional magnetic order sets in. However, even for temperatures above this ordering temperature interchain couplings can have a significant effect which has to be taken into account.

In what follows, we will study the anisotropic spin-1/2 Heisenberg chain (XXZ model) with $N$ 
sites and $\mathrm{OBCs}$

$$
H=J \sum_{j=1}^{N-1}\left[S_{j}^{x} S_{j+1}^{x}+S_{j}^{y} S_{j+1}^{y}+\Delta S_{j}^{z} S_{j+1}^{z}\right]-h \sum_{j=1}^{N} S_{j}^{z} .
$$

Here $J$ is the exchange constant and $h$ is the applied magnetic field. Although exchange anisotropies due to spin-orbit coupling are usually rather small so that experimentally only the isotropic case $\Delta=1$ is relevant, the additional parameter $\Delta$ is useful for the field theoretical calculations in section 3. There are two important consequences of the OBCs. First, expectation values of local operators become position dependent because translational invariance is broken. In the following we will, in particular, study the local susceptibility defined as

$$
\chi_{j}=\frac{\partial}{\partial h}\left\langle S_{j}^{z}\right\rangle_{h=0}=\frac{1}{T}\left\langle S_{j}^{z} S_{\mathrm{tot}}^{z}\right\rangle_{h=0}
$$

where $S_{\text {tot }}^{z}=\sum_{j} S_{j}^{z}$. Second, there are well defined boundary contributions to all thermodynamic quantities. For $N \rightarrow \infty$ the total free energy $F$ is, for example, given by

$$
F=N f_{\text {bulk }}+F_{\mathrm{B}}+\mathcal{O}(1 / N)
$$

where $F_{\mathrm{B}}$ is the boundary free energy. Similarly, one can define a boundary susceptibility $\chi_{\mathrm{B}}\left[18^{-}\right.$ $22]$. These boundary or surface terms will be studied here as well. The local susceptibility defined in equation (1.2) can be related to the boundary susceptibility by

$$
\chi_{\mathrm{B}}=\lim _{N \rightarrow \infty}\left(\sum_{j=1}^{N} \chi_{j}-N \chi_{\mathrm{bulk}}\right)
$$

where $\chi_{\text {bulk }}$ is the bulk susceptibility defined analogously to the bulk free energy in equation (1.3).

For the interchain couplings we will consider two different cases relevant for many materials. One is a simple ladder-like antiferromagnetic coupling between neighboring chains as, for example, in $\mathrm{Sr}_{2} \mathrm{CuO}_{3}$ as well as in many other spin chain compounds. This case is shown in figure $1 \mathrm{~b}$. The other is a zigzag ferromagnetic coupling between neighboring chains. This kind of interchain coupling is sketched in figure $1 \mathrm{c}$ and is relevant, for example, for $\mathrm{SrCuO}_{2}$ [1].

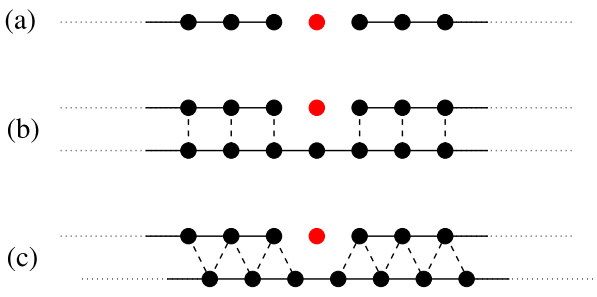

Figure 1. A non-magnetic impurity in (a) a single spin chain, (b) in a spin chain coupled to a neighboring chain by a ladder-like interchain coupling, and (c) in a spin chain coupled in a zigzag fashion to a neighboring chain.

To provide an intuitive picture we start with some numerical results for semi-infinite spin chains with geometries as shown in figure 1 obtained by the density matrix renormalization group applied to transfer matrices (TMRG) in section 2. In section 3 we will present the field theory for finite Heisenberg chains with OBCs at finite temperatures. In section 4 we use the results obtained in the previous section to calculate the susceptibility as an ensemble average over finite spin chain segments and show that the obtained results are in good agreement with experimental measurements. In section 5 we derive, in a similar fashion, the nuclear magnetic resonance (NMR) spectrum and show that it provides information about the interchain couplings. Finally, we give a brief summary and present some conclusions. 


\section{Numerical results}

A method particularly suited to the calculation of the thermodynamic properties of onedimensional systems is the density matrix renormalization group applied to transfer matrices (TMRG) [20-28]. To this end, the one-dimensional quantum system is mapped onto a two-dimensional classical system by a Trotter-Suzuki decomposition [29-31]. Then, the additional dimension corresponds to the inverse temperature $\beta$. For the classical model, a transfer matrix is defined which evolves along the spatial direction. Importantly, one can show that even for a critical system there is always a gap at finite temperatures between the leading eigenvalue $\Lambda_{0}$ and the next-leading eigenvalues $\Lambda_{\alpha}$ of the transfer matrix $T$ with $\xi_{\alpha}^{-1}=\ln \left|\Lambda_{0} / \Lambda_{\alpha}\right|$ defining a correlation length. This makes it possible to perform the thermodynamic limit, i. e., system size $N \rightarrow \infty$, exactly. With the TMRG one can treat impurity problems [21,22,32] as well as frustrated systems [33] making it an ideal numerical tool to study the problem considered here.

We will start by investigating the local susceptibility as defined in equation (1.2) for a semiinfinite chain. By this we mean a chain which is infinitely long but has one end with OBCs. To obtain $\chi_{j}$ we calculate the local magnetization $\left\langle S_{j}^{z}\right\rangle$ for small magnetic fields $h / J \sim 10^{-2}$ and take a numerical derivative. Within the transfer matrix formalism the local magnetization is given by

$$
\lim _{N \rightarrow \infty}\left\langle S_{j}^{z}\right\rangle=\frac{\left\langle\Psi_{L}^{0}\left|T\left(S^{z}\right) T^{j-1} \widetilde{T}\right| \Psi_{R}^{0}\right\rangle}{\Lambda_{0}^{j}\left\langle\Psi_{L}^{0}|\widetilde{T}| \Psi_{R}^{0}\right\rangle}
$$

Here $\widetilde{T}$ is a modified transfer matrix containing the broken bond, $T\left(S^{z}\right)$ is the transfer matrix with the operator $S^{z}$ included, and $\left|\Psi_{R}^{0}\right\rangle\left(\left\langle\Psi_{L}^{0}\right|\right)$ are the right (left) eigenstates belonging to the largest eigenvalue $\Lambda_{0}$, respectively. Far away from the boundary $\left\langle S_{j}^{z}\right\rangle$ becomes a constant, the bulk magnetization

$$
m=\lim _{j \rightarrow \infty} \lim _{N \rightarrow \infty}\left\langle S_{j}^{z}\right\rangle=\lim _{j \rightarrow \infty} \frac{\sum_{n}\left\langle\Psi_{L}^{0}\left|T\left(S^{z}\right) T^{j-1}\right| \Psi_{R}^{n}\right\rangle\left\langle\Psi_{L}^{n}|\widetilde{T}| \Psi_{R}^{0}\right\rangle}{\Lambda_{0}^{j}\left\langle\Psi_{L}^{0}|\widetilde{T}| \Psi_{R}^{0}\right\rangle}=\frac{\left\langle\Psi_{L}^{0}\left|T\left(S^{z}\right)\right| \Psi_{R}^{0}\right\rangle}{\Lambda_{0}}
$$

By taking again a numerical derivative with respect to a small magnetic field, we can obtain the bulk susceptibility $\chi_{\text {bulk }}$ from $(2.2)$.

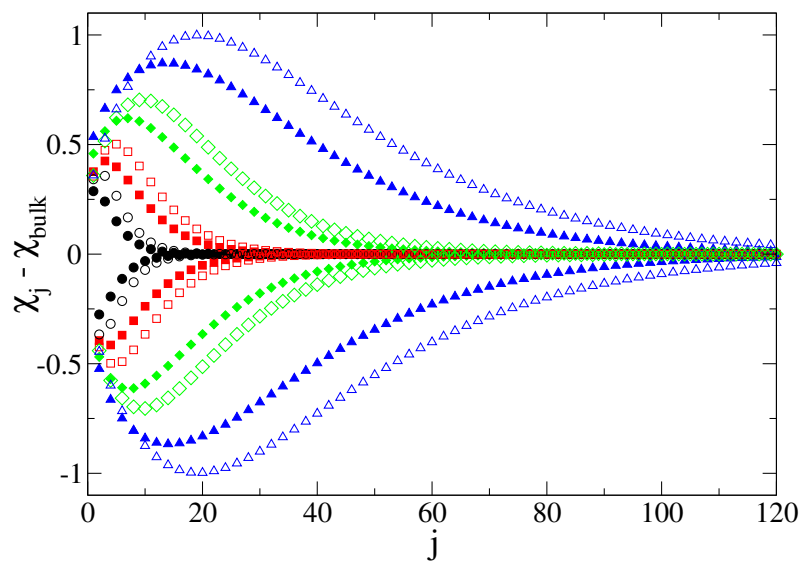

Figure 2. $\chi_{j}-\chi_{\text {bulk }}$ for a semi-infinite chain with $\Delta=1$ at temperatures $T / J=$ $0.2,0.1,0.05,0.025$. The numerical TMRG data (closed symbols) are compared to the field theory formula (3.23) from section 5 (open symbols).

In figure 2 the susceptibility profile $\chi_{j}-\chi_{\text {bulk }}$ for a single semi-infinite chain with $\Delta=1$ is shown. As might be expected, the boundary induces Friedel-like oscillations which become larger with decreasing temperature. Interestingly, at low temperatures the oscillations first increase and reach a maximum, before decaying at large distances. This phenomenon has been first studied by 
Eggert and Affleck in [34] and we will rederive their field theory result as a special case of our more general considerations in section 3 .

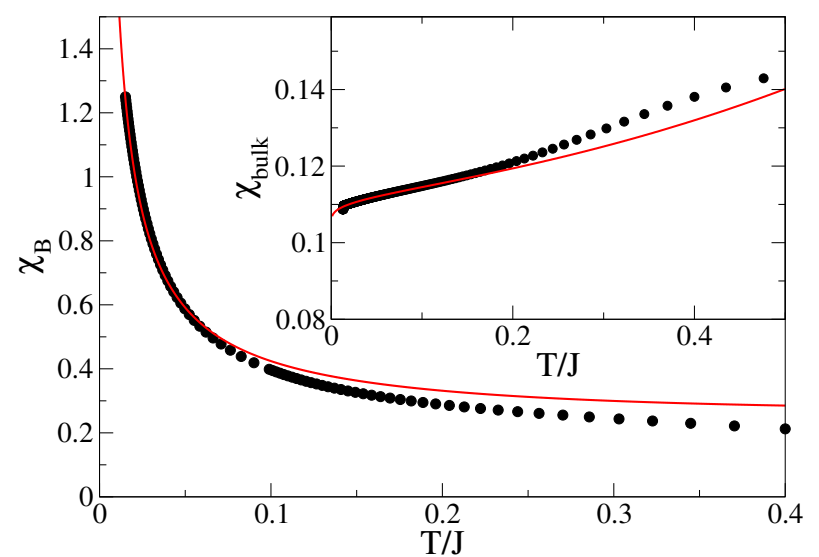

Figure 3. The boundary susceptibility $\chi_{\mathrm{B}}$ for $\Delta=1$ as obtained by TMRG (symbols) compared to the field theory result (3.20) (line) derived in section 3 which is valid at low temperatures. Inset: Numerical results for the bulk susceptibility $\chi_{\text {bulk }}$ for $\Delta=1$ (symbols) compared to the field theory formula (3.19) (line).

Next, we consider the boundary susceptibility which we can easily obtain from the susceptibility profile using equation (1.4). The result is shown in figure 3. At low temperatures it was shown analytically that $\chi_{\mathrm{B}} \sim\left[T \ln \left(T_{0} / T\right)\right]^{-1}$ with a known constant $T_{0}$ [17-19]. The bulk susceptibility, on the other hand, behaves like $\chi_{\text {bulk }} \sim$ const $+\ln ^{-1}\left(T_{0} / T\right)$ [12], i. e., it goes to a finite value at $T=0$ with infinite slope. We will come back to this in section 3 but let us notice here that the numerical data are well described by the field theory.

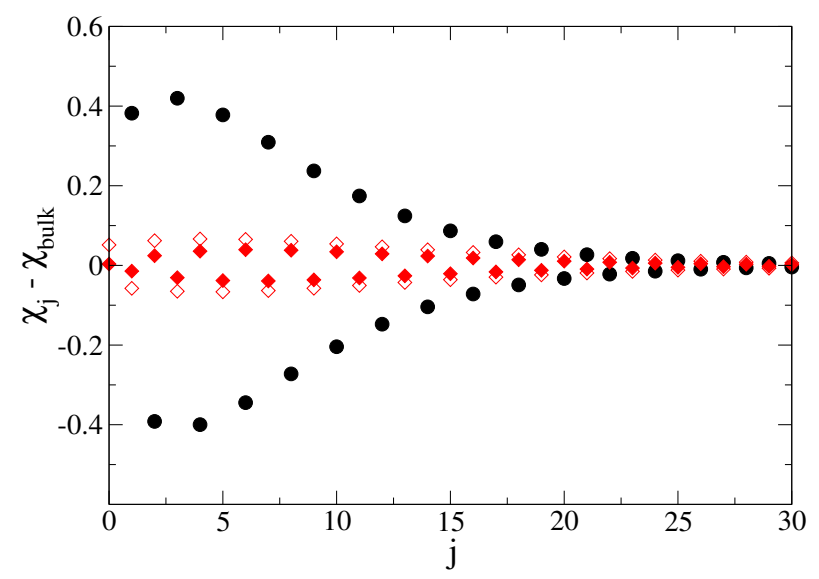

Figure 4. $\chi_{j}-\chi_{\text {bulk }}$ for $\Delta=1, J_{\perp}=0.03 J$ and $T=0.09 J$ and a ladder-like interchain coupling as shown in figure $1 \mathrm{~b}$. One of the chains has a non-magnetic impurity at site $j=0$ (circles) whereas the other one (diamonds) is infinitely long and does not have any impurities. The numerical TMRG data (closed symbols) are compared to the field theory formula (5.4) from section 5 (open symbols).

Finally, we have also calculated susceptibility profiles for the cases of coupled chains shown in figure $1 \mathrm{~b}$ and c. A ladder-like coupling of neighboring chains is, for example, realized in $\mathrm{Sr}_{2} \mathrm{CuO}_{3}$ [1]. In figure 4 it is shown that in this case an impurity in one chain has also a significant effect on a neighboring chain without impurities. The Friedel-like oscillations are reflected in the impurity- 
free chain due to the interchain couplings. Clearly, the size of the reflected Friedel-like oscillations will depend on the ratio $J_{\perp} / T$. However, this ratio is not the only relevant factor. The geometry of the interchain couplings plays an important role as well. In $\mathrm{SrCuO}_{2}$, neighboring chains are coupled by a ferromagnetic zigzag-type coupling. In this case an impurity in one chain leaves a neighboring chain almost unaffected even if the temperature $T \sim J_{\perp}$ as is shown in figure 5 . In section 5 we will show that these differences can be easily understood if one starts from the field theory results for a single chain derived in the next section and takes the interchain couplings into account perturbatively.

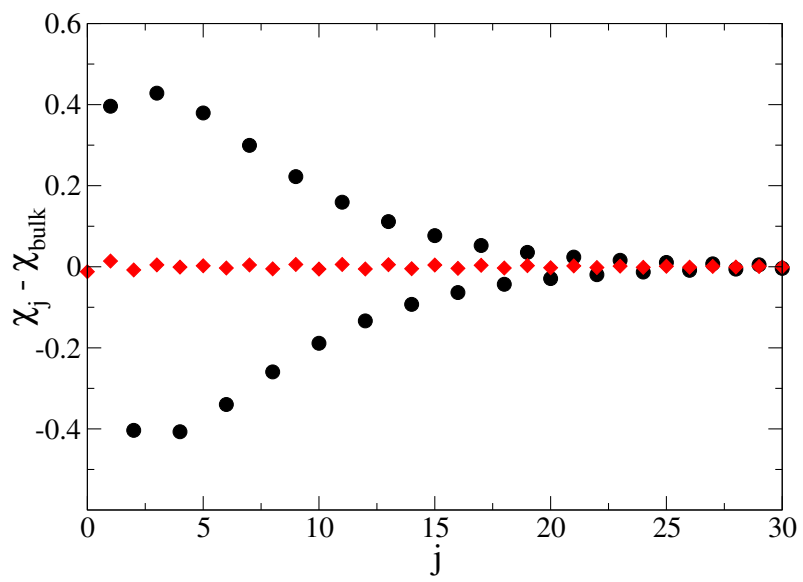

Figure 5. $\chi_{j}-\chi_{\text {bulk }}$ for $\Delta=1, J_{\perp}=-0.1 J$ and $T=0.09 J$ with a zigzag interchain coupling as shown in figure 1c. One of the chains has a non-magnetic impurity at site $j=0$ (circles) whereas the other one (diamonds) is infinitely long and does not have any impurities. For this case the field theory predicts that there are no oscillations, $\chi_{j}-\chi_{\text {bulk }} \approx 0$, in the infinitely long chain.

\section{Field theory for finite spin chains}

In the limit of low temperatures and large chain length, the XXZ model (1.1) can be represented by a field theory. The main step is a linearization of the dispersion around the two Fermi points. A certain linear combination of particle-hole excitations around a Fermi point constitutes a collective bosonic mode described by the Luttinger liquid Hamiltonian

$$
H=\frac{v}{2} \int_{0}^{L+a} \mathrm{~d} x\left[\Pi^{2}+\left(\partial_{x} \Phi\right)^{2}\right]-h \sqrt{\frac{K}{2 \pi}} \int_{0}^{L+a} \mathrm{~d} x \partial_{x} \Phi,
$$

where $v$ is the spin velocity, $L=N a$, and $a$ is the lattice constant. The bosonic field $\Phi$ obeys the standard commutation rule $\left[\Phi(x), \Pi\left(x^{\prime}\right)\right]=\mathrm{i} \delta\left(x-x^{\prime}\right)$ with $\Pi=v^{-1} \partial_{t} \Phi$. The Luttinger parameter $K$ is a function of the anisotropy $\Delta$ and can be determined exactly by Bethe ansatz with $K=1$ at the isotropic point. The spin operators can be directly expressed in terms of the boson $\Phi$, in particular, we have

$$
S_{j}^{z} \approx \sqrt{\frac{K}{2 \pi}} \partial_{x} \Phi+c(-1)^{j} \cos \sqrt{2 \pi K} \Phi
$$

at zero magnetic field. Here $c$ is an amplitude which can also be obtained exactly [35-37]. The separation of $S_{j}^{z}$ into a uniform and a staggered part at low energies can be understood as follows. In the equivalent spinless fermion representation of the XXZ model, obtained by a Jordan-Wigner transformation, the $S^{z}$ operator becomes the density operator. Due to the linearization of the dispersion the electron and the hole can live either at the same Fermi point and have, therefore, small momentum or they can be situated at different Fermi points in which case the associated 
momentum is $2 k_{\mathrm{F}}$. Zero magnetic field corresponds to half-filling for the spinless fermions. Thus, $2 k_{\mathrm{F}}=\pi$ and we obtain the staggered contribution in (3.2). This also means that the local susceptibility defined in equation (1.2) can be separated at low temperatures into a uniform and a staggered part

$$
\chi_{j}=\chi^{\mathrm{uni}}+(-1)^{j} \chi_{j}^{\mathrm{st}}
$$

In a bulk susceptibility measurement, the staggered part does not contribute. This part is, however, measurable in probes of the local magnetism as, for example, in NMR. We will come back to this point in section 5 .

If one is only interested in correlation functions for infinite system size at finite temperatures or correlation functions for finite systems at zero temperature, one can make use of the conformal invariance of the field theory. It is then sufficient to calculate the correlation at zero temperature and infinite system size and use a conformal mapping from the complex plane onto a cylinder with the circumference corresponding either to inverse temperature or system size. Here, we are, however, interested in the thermodynamics of finite chains and we will, therefore, have to use an explicit mode expansion

$$
\Phi(x=j a, t)=\sqrt{\frac{\pi}{8 K}}+\sqrt{\frac{2 \pi}{K}} S_{\text {tot }}^{z} \frac{j}{N+1}+\sum_{n=1}^{\infty} \frac{\sin (\pi n j /(N+1))}{\sqrt{\pi n}}\left(\mathrm{e}^{-i \frac{\pi n v t}{L+a}} b_{n}+\mathrm{e}^{i \frac{\pi n v t}{L+a}} b_{n}^{\dagger}\right)
$$

which incorporates the OBCs. Here $b_{n}$ is a bosonic annihilation operator. Equation (3.4) is a discrete version of the mode expansions used in $[13,16]$ with $x=j a$ becoming a continuous coordinate for $a \rightarrow 0, N \rightarrow \infty$ with $L=N a$ fixed. Using this mode expansion, the local observables respect the discrete lattice symmetry $j \rightarrow N+1-j$ corresponding to a reflection at the central bond (site) for $N$ even (odd), respectively. The sites 0 and $N+1$ are added to model (1.1) and we demand that the spin density should vanish at these sites. Therefore, the upper boundary for the integrals in (3.1) is $L+a$. The zero mode part (first two terms of equation (3.4)) fulfills $\sum_{j} S_{j}^{z} \approx \sqrt{\frac{K}{2 \pi}} \int_{0}^{L+a} \partial_{x} \Phi \equiv S_{\text {tot }}^{z}$ and the oscillator part (last term of equation (3.4)) vanishes for $j=0, N+1$ as required.

For the free boson model, the uniform part of the susceptibility can easily be calculated and is given by $[13]$

$$
\chi_{0}^{\text {uni }}=-\left.\frac{\partial^{2}}{\partial h^{2}}\right|_{h=0} f_{0}=\frac{1}{N T} \frac{\sum_{S_{z}} S_{z}^{2} \mathrm{e}^{-\frac{\pi v}{K(L+a) T} S_{z}^{2}}}{\sum_{S_{z}} \mathrm{e}^{-\frac{\pi v}{K(L+a) T} S_{z}^{2}}}=-\left.\frac{1}{4 N T} \frac{\partial^{2}}{\partial u^{2}}\right|_{u=0} \ln \theta\left(\mathrm{e}^{-\frac{\pi v}{K(L+a) T}}, u\right) .
$$

Here $\theta(q, u)$ is the elliptic theta function of the third kind $\theta=\theta_{3}(q, u)=\sum_{n=-\infty}^{\infty} q^{n^{2}} \mathrm{e}^{i 2 n u}$ for integer $S_{z}$ (even $N$ ) and of the second kind $\theta=\theta_{2}(q, u)=\sum_{n=-\infty}^{\infty} q^{(n+1 / 2)^{2}} \mathrm{e}^{i(2 n+1) u}$ for halfinteger $S_{z}($ odd $N)$. Note, that $\chi_{0}^{\text {uni }}$ has a simple scaling form as a function of $N T$. The lattice parameter $a$ appears here due to the OBCs. It leads to a boundary correction which we will consider later. First, we set $a \equiv 0$ in (3.5) and concentrate on the following limiting cases

$$
\chi_{0}^{\text {uni }}= \begin{cases}\frac{2}{T N} \exp \left[-\frac{\pi v}{K L T}\right] & N T / v \rightarrow 0, N \text { even }, \\ \frac{1}{4 T N} & N T / v \rightarrow 0, N \text { odd }, \\ \frac{K}{2 \pi v} & N T / v \rightarrow \infty .\end{cases}
$$

The Curie-like divergence for $N$ odd is caused by the degeneracy of the ground state $S_{\text {tot }}^{z}= \pm 1 / 2$. At low temperatures the whole chain, therefore, behaves like a single spin. For $N$ even, on the other hand, the ground state is a singlet, $S_{\text {tot }}^{z}=0$. At low temperatures, the chain becomes locked in this state leading to an exponentially small susceptibility. In the thermodynamic limit, $N T / v \rightarrow \infty$, the susceptibility within the free boson approximation is just a constant.

The staggered part of the susceptibility, $\chi_{j}^{\text {st }}$, for a finite chain with OBCs has been calculated in [37]. It is given by

$$
\chi_{j}^{\mathrm{st}}=-\frac{c}{T}\left(\frac{\pi}{N+1}\right)^{K / 2} \frac{\eta^{3 K / 2}\left(\mathrm{e}^{-\frac{\pi v}{T L}}\right)}{\theta_{1}^{K / 2}\left(\frac{\pi j}{N+1}, \mathrm{e}^{-\frac{\pi v}{2 T L}}\right)} \frac{\sum_{m} m \sin [2 \pi m j /(N+1)] \mathrm{e}^{-\pi v m^{2} /(K L T)}}{\sum_{m} \mathrm{e}^{-\pi v m^{2} /(K L T)}} .
$$


Here $\eta(x)$ is the Dedekind eta-function and $\theta_{1}(u, q)$ the elliptic theta-function of the first kind. The summation index $m$ runs over all integers (half-integers) for $N$ even (odd), respectively. In the thermodynamic limit, $N \rightarrow \infty$, we can simplify our result and obtain

$$
\chi_{j}^{\mathrm{st}}=\frac{c K}{v} \frac{x}{\left[\frac{v}{\pi T} \sinh \left(\frac{2 \pi T x}{v}\right)\right]^{K / 2}}
$$

with $x=j a$. This agrees for the isotropic Heisenberg case, $K=1$, with the result in [34]. The amplitude $c$, first introduced in equation (3.2), can be determined with the help of the Bethe ansatz along the lines of [36]. This leads to $c=\sqrt{A_{z} / 2}$ with $A_{z}$ as given in equation (4.3) of [36]. The formulas (3.7) and (3.8) are, therefore, parameter free.

To find the boundary contributions, such as the boundary free energy defined in equation (1.3), one has to go beyond the free boson model (3.1). When deriving the low-energy effective theory for the XXZ model, one finds in addition to the free boson model (3.1) infinitely many irrelevant terms. These terms either stem from band curvature (corrections to the linear dispersions around the Fermi points) or from the interaction term. For $\Delta$ close to 1 the leading irrelevant term is given by

$$
\delta H=\lambda \int_{0}^{L+a} \mathrm{~d} x \cos (\sqrt{8 \pi K} \phi) .
$$

It is the bosonized version of Umklapp scattering where two left moving electrons get scattered to right movers or vice versa interchanging a reciprocal lattice vector. This term becomes relevant for $\Delta>1$ and is responsible for the opening of an excitation gap in this regime. For the isotropic case, $\Delta=1$, Umklapp scattering is marginally irrelevant and leads to important corrections to the results obtained for the free boson model. Due to the integrability of the XXZ model the amplitude $\lambda$ of the Umklapp term can be obtained exactly as well [38]. In [16,17] the free energy and the susceptibility corrections to the free boson result up to the first order in the Umklapp scattering have been calculated. For the susceptibility the following correction was obtained

$$
\delta \chi_{1}^{\mathrm{uni}}=\frac{2 \lambda}{T^{2}}\left(\frac{\pi}{N}\right)^{2 K} \eta^{6 K}\left(\mathrm{e}^{-\frac{\pi v}{T L}}\right) \int_{0}^{1 / 2} \mathrm{~d} y \frac{g_{0}\left(y, \mathrm{e}^{-\frac{\pi v}{K L T}}\right)}{\theta_{1}^{2 K}\left(\pi y, \mathrm{e}^{-\frac{\pi v}{2 T L}}\right)}
$$

with

$$
g_{0}(y, q)=-\frac{\sum_{S_{z}} S_{z}^{2} \cos \left(4 \pi S_{z} y\right) q^{S_{z}^{2}}}{\sum_{S_{z}} q^{S_{z}^{2}}}+\frac{\left(\sum_{S_{z}} \cos \left(4 \pi S_{z} y\right) q^{S_{z}^{2}}\right)\left(\sum_{S_{z}} S_{z}^{2} q^{S_{z}^{2}}\right)}{\left(\sum_{S_{z}} q^{S_{z}^{2}}\right)^{2}}
$$

In addition, there is also a boundary correction related to the parameter $a$ in (3.5). Expanding in this parameter to the lowest order we find

$$
\delta \chi_{2}^{\mathrm{uni}}=\frac{\pi v a}{K T^{2} L^{3}} g_{2}\left(\mathrm{e}^{-\frac{\pi v}{K L T}}\right)
$$

where

$$
g_{2}(q)=\frac{\sum_{S_{z}} S_{z}^{4} q^{S_{z}^{2}}}{\sum_{S_{z}} q^{S_{z}^{2}}}-\frac{\left(\sum_{S_{z}} S_{z}^{2} q^{S_{z}^{2}}\right)^{2}}{\left(\sum_{S_{z}} q^{S_{z}^{2}}\right)^{2}}
$$

$a$ plays the role of a lattice constant and its value can be determined for $\Delta<1(K>1)$ by the Bethe ansatz and is given by $[20,21]$

$$
a=2^{-1 / 2} \sin [\pi K /(4 K-4)] / \cos [\pi /(4 K-4)] .
$$

The uniform part of the susceptibility of a finite chain is, therefore, given by $\chi^{\mathrm{uni}}(L, T)=\chi_{0}^{\mathrm{uni}}(a \equiv$ $0)+\delta \chi_{1}^{\text {uni }}+\delta \chi_{2}^{\text {uni }}$. The corrections to the uniform zeroth order susceptibility $\chi_{0}^{\text {uni }}(a \equiv 0)$ are 
important here because in the thermodynamic limit they give the boundary susceptibility $\chi_{\mathrm{B}}$. In this limit $g_{2}\left(\mathrm{e}^{-\pi v /(K L T)}\right) \rightarrow K^{2} T^{2} L^{2} /\left(2 \pi^{2} v^{2}\right)$ and therefore

$$
\chi_{B, 1}=\lim _{L \rightarrow \infty} L \chi_{2}^{\text {uni }}=\frac{K a}{2 \pi v} .
$$

This is just a constant contribution to the boundary susceptibility. Much more important is the boundary contribution stemming from $\delta \chi_{1}^{\text {uni }}$. Here we find

$$
\chi_{B, 2}=\lim _{L \rightarrow \infty} L \chi_{1}^{\mathrm{uni}}=-\lambda\left(\frac{K}{v}\right)^{2} B(K, 1-2 K)\left[\pi^{2}-2 \psi^{\prime}(K)\right]\left(\frac{2 \pi T}{v}\right)^{2 K-3}
$$

with $B(x, y)=\Gamma(x) \Gamma(y) / \Gamma(x+y), \psi^{\prime}(x)=\mathrm{d} \psi(x) / \mathrm{d} x$, and $\psi(x)$ being the digamma function. Note that for $1<K<3 / 2(1 / 2<\Delta<1)$ the boundary spin susceptibility $\chi_{\mathrm{B}}$ shows a divergent behavior $\sim 1 / T^{3-2 K}$ as temperature decreases.

\subsection{The isotropic point}

At the isotropic point, Umklapp scattering becomes marginal and simple perturbation theory is no longer sufficient. In this case we have to replace the Umklapp scattering amplitude by a running coupling constant $g(L, T)$ which obeys a known set of renormalization group equations [38]

$$
1 / g+\ln (g) / 2=\ln \left(\sqrt{2 / \pi} \mathrm{e}^{1 / 4+\gamma} \min [L, v / T]\right) .
$$

Here $\gamma \approx 0.577$ is Euler's constant and for the isotropic case considered here, the spin velocity is $v=J \pi / 2$. Then, the uniform susceptibility is given by

$$
\chi^{\text {uni }}(N, T)=\chi_{0}^{\text {uni }}+\delta \chi_{1}^{\text {uni }}
$$

where $K \rightarrow 1+g(L, T) / 2$ in the exponentials of (3.5) and $\lambda \rightarrow g(L, T) / 4$ in (3.10). In this case the parameter $a$ in (3.5) is not determined by (3.14) and has to be used as a fitting parameter.

In the thermodynamic limit we can again split the susceptibility (3.18) into a bulk and a boundary part. For the bulk susceptibility this yields the result first derived by Lukyanov [38]

$$
\chi_{\mathrm{bulk}}=\frac{1}{\pi^{2}}\left(1+\frac{g(T)}{2}+\frac{3 g^{3}(T)}{32}+\frac{\sqrt{3}}{\pi} T^{2}\right) .
$$

Here we have also added the $g^{3}$ correction from Umklapp scattering as well as a $T^{2}$-term which stems from irrelevant operators with scaling dimension 4 describing the band curvature. The running coupling constant $g(T)$ is given by (3.17) with $L=\infty$. In the inset of figure 3 this formula is displayed in comparison with the numerical results. For the boundary susceptibility, on the other hand, we find

$$
\chi_{\mathrm{B}}=\frac{a}{\pi^{2}}+\frac{g}{12 T}+\frac{g^{2}}{8 T}\left(0.66-\frac{\Psi^{\prime \prime}(1)}{\pi^{2}}\right)+\mathcal{O}\left(g^{3}\right),
$$

where we included the second order corrections in $g$ as derived in [17]. The comparison of this formula with $a=1.5$ and numerical results is shown in the main panel of figure 3 .

Finally, the result for the staggered part of the susceptibility given in equation (3.7) has to be modified at the isotropic point. Here we find [37]

$$
\chi_{j}^{\mathrm{st}}=-\frac{1}{\left(2 \pi^{3} \tilde{g}\right)^{1 / 4} T}\left(\frac{\pi}{N+1}\right)^{1 / 2} \frac{\eta^{3 / 2}\left(\mathrm{e}^{-\frac{\pi v}{T L}}\right)}{\theta_{1}^{1 / 2}\left(\frac{\pi j}{N+1}, \mathrm{e}^{-\frac{\pi v}{2 T L}}\right)} \frac{\sum_{m} m \sin [2 \pi m j /(N+1)] \mathrm{e}^{-\pi v m^{2}(1-\tilde{g}) /(L T)}}{\sum_{m} \mathrm{e}^{-\pi v m^{2}(1-\tilde{g}) /(L T)}}
$$

Now the running coupling constant $\tilde{g}$ also depends on the distance of site $j$ from the boundary and is given by

$$
1 / \tilde{g}+\ln (\tilde{g}) / 2=\ln \left(\min \left[C_{0} x, C_{0}(L-x), \sqrt{\pi / 2} \mathrm{e}^{1 / 4+\gamma} / T\right]\right)
$$


where the constant $C_{0}$ is not known and has to be used as a fitting parameter. Note, however, that for low temperatures and $x, L-x \gg 1$ the value of $C_{0}$ becomes irrelevant and our result for $\chi_{j}$, therefore, is again parameter-free. If we consider the thermodynamic limit of equation (3.21) we find

$$
\chi_{j}^{\mathrm{st}}=\left(\frac{2^{3}}{\pi^{7} \tilde{g}}\right)^{1 / 4} \frac{1}{1-\tilde{g}} \frac{x}{\sqrt{\frac{1}{2 T} \sinh (4 T x)}} .
$$

A comparison of this formula with numerical data is shown in figure 2. The agreement is good but not perfect. The main problem here is that the temperature and the length scale set by the distance from the boundary are competing. The renormalization group equations, however, cannot be solved with both scales present. The formula (3.22) is derived in the limit when only one of those scales matter and the corrections can be significant if this is not the case.

\section{The averaged susceptibility and a comparison with experimental data}

In an actual crystal we have imperfections and impurities which limit the length of a spin chain segment. It is important to emphasize again that any weakening of a link is a relevant perturbation in the renormalization group sense. It is, therefore, expected to be a good approximation to assume that at low temperatures we have spin chain segments with open boundary conditions. As long as we do not know in detail how these defects are distributed it seems reasonable to assume a Poisson distribution, i. e., the probability of having an impurity at site $j$ is not effected by the other impurities. We might, however, expect this assumption to break down for large impurity concentrations where some sort of impurity order might set in. Using a Poisson distribution we have a normalized probability $P(N)=p(1-p)^{N}$ to find a chain segment with $N$ sites if the impurity concentration is $p$. Any measurement corresponds to taking an average over this ensemble of chain segments. For the susceptibility, for example, we find

$$
\chi_{p}=p^{2} \sum_{N} N(1-p)^{N} \chi(N) .
$$

Note that in a bulk measurement only the uniform part of the susceptibility contributes. The staggered part cancels out. Using the formula (3.18) we can immediately calculate the average susceptibility $\chi_{p}$. It is, however, very useful and instructive to derive a much simpler approximate formula. To this end, we notice that we have two different regimes for a finite spin chain. If the temperature is larger than the finite size gap, $T / J>1 / N$, we can approximate $\chi(N) \approx$ $\chi_{\text {bulk }}+\chi_{\mathrm{B}} / N$. If, on the other hand, $T / J<1 / N$ we expect to see more or less the ground state properties of the finite chain. This means, according to equation (3.6), that there will be no contribution when the chain length is even while $\chi(N) \approx 1 /(4 T N)$ if the chain length is odd. We can, therefore, introduce a length $N_{c}=\gamma J / T$ where this crossover occurs. Here $\gamma$ is a parameter which we expect to be of the order of 1 . For the average susceptibility we can, therefore, write

$$
\begin{aligned}
\chi_{p} & \approx \frac{p^{2}}{4 T} \sum_{N \text { odd }}^{N_{c}}(1-p)^{N}+p^{2} \sum_{N=N_{c}}^{\infty}\left(N \chi_{\text {bulk }}+\chi_{\mathrm{B}}\right)(1-p)^{N} \\
& =\frac{p}{4 T} \frac{1-p}{2-p}\left(1-(1-p)^{\gamma / T}\right)+(1-p)^{\gamma / T}\left[\left(1-p+\frac{p \gamma}{T}\right) \chi_{\text {bulk }}+p \chi_{\mathrm{B}}\right]
\end{aligned}
$$

where $\chi_{\text {bulk }}$ is given by equation (3.19) and $\chi_{\mathrm{B}}$ by equation (3.20).

An interesting experiment has been performed by Kojima et al. [5] where Palladium (Pd) has been doped into the spin chain compound $\mathrm{Sr}_{2} \mathrm{CuO}_{3}$. The $\mathrm{Pd}$ ions replace the $\mathrm{Cu}$ ions, act as nonmagnetic impurities, and cut the chain into finite segments. A complication in the analysis of this experiment arises because even the undoped $\mathrm{Sr}_{2} \mathrm{CuO}_{3}$ samples already have a substantial amount of chain breaks. It is believed that this is mainly a consequence of excess oxygen, i. e., we are in reality dealing with $\mathrm{Sr}_{2} \mathrm{CuO}_{3+\delta}$. In a simple picture, an excess oxygen ion pulls two electrons out of the copper chain. If these holes are relatively immobile this also corresponds to a chain break. 
In the comparison of the experimental data for $\mathrm{Sr}_{2} \mathrm{Cu}_{1-x} \mathrm{Pd}_{x} \mathrm{O}_{3+\delta}$ and formula (4.2) shown in figure 6 we, therefore, use $p$ as an effective impurity concentration incorporating both the chain breaks due to excess oxygen and due to the non-magnetic Pd ions. Furthermore, we have subtracted a constant contribution $\chi_{\text {const }}$ from the experimental data which is expected to be present due to core diamagnetism and Van Vleck paramagnetism. The values for $p$ and $\chi_{\text {const }}$ which yield the best fit of the experimental data are shown in table 1. For Pd concentrations of $x=0.5 \%$ and

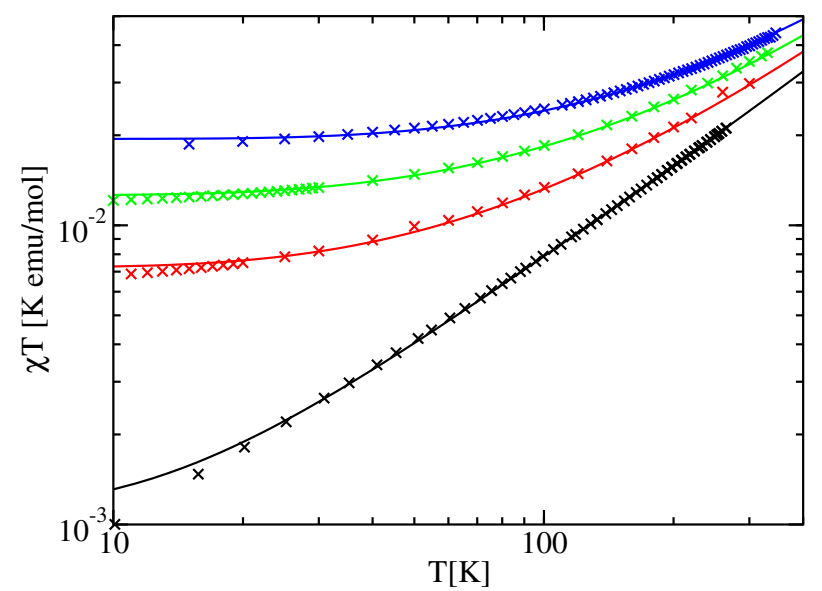

Figure 6. Measured susceptibility $T\left(\chi-\chi_{\text {const }}\right)$ for $\mathrm{Sr}_{2} \mathrm{Cu}_{1-x} \mathrm{Pd}_{x} \mathrm{O}_{3+\delta}$ with impurity concentrations $x=0 \%, 0.5 \%, 1 \%, 3 \%$ (crosses from bottom to top) from [5]. Here a constant $\chi_{\text {const }}$ as given in table 1 has been subtracted from the experimental data. Subsequent curves are shifted by $5 \times 10^{-3}$. For comparison theoretical results according to (4.2) with $\gamma=1$ are shown with an effective impurity concentration $p$ as given in table 1 .

Table 1. Concentration $x$ of $\mathrm{Pd}$ ions in experiment compared to chain break concentration $p$ and constant contribution $\chi_{\text {const }}$ yielding the best theoretical fit. The first line corresponds to the "as grown" sample of $\mathrm{Sr}_{2} \mathrm{CuO}_{3+\delta}$ from [1].

\begin{tabular}{c|c|c}
$x$ (Exp.) & $p$ (Theory) & $\chi_{\text {const }}[\mathrm{emu} / \mathrm{mol}]$ \\
\hline \hline 0.0 & 0.006 & $-7.42 \times 10^{-5}$ \\
0.005 & 0.012 & $-7.7 \times 10^{-5}$ \\
0.01 & 0.014 & $-7.5 \times 10^{-5}$ \\
0.03 & 0.024 & $-7.5 \times 10^{-5}$
\end{tabular}

$x=1 \%$ the obtained values for $p$ are consistent with the picture of having a certain amount of chain breaks due to excess oxygen already in the undoped compound. For $x=3 \%$, however, this picture seems to fail. Reasons for this could be either on the experimental side (perhaps not all $\mathrm{Pd}$ ions really go into the sample, or some go in interstitially) or in the theoretical description. If, for example, the Pd ions tend to cluster above a certain concentration, then our assumption of a Poisson distribution becomes incorrect.

\section{The Knight shift and the role of interchain couplings}

As already mentioned, the staggered part of the susceptibility cannot be observed in a bulk measurement. This, however, is possible by NMR because here the resonance frequency gets shifted by the local magnetic field. This so-called Knight shift $K_{j}$ can, therefore, be directly related to the 
local susceptibility. For a chain of length $N$ it is given by

$$
K_{j}^{(N)}=\left(\gamma_{e} / \gamma_{n}\right) \sum_{j^{\prime}} A^{j-j^{\prime}} \chi_{j^{\prime}}^{(N)}
$$

where $\gamma_{e}\left(\gamma_{n}\right)$ is the electron (nuclear) gyromagnetic ratio, respectively and $A_{j-j}$ the hyperfine coupling tensor. It is usually sufficient to take only $A^{0}$ and $A^{ \pm 1}$ into account because of the shortrange nature of the hyperfine interaction.

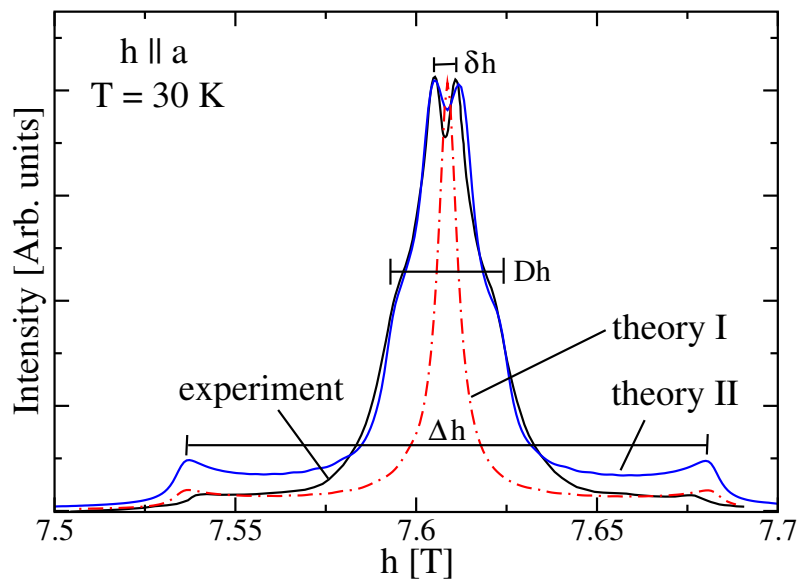

Figure 7. $\mathrm{NMR}$ spectrum for $\mathrm{Sr}_{2} \mathrm{CuO}_{3}$ at $T=30 \mathrm{~K}$ taken from [6]. In comparison the theoretical results for a single chain with a Poisson distribution of chain breaks (theory I) and for the case where also interchain couplings $\left(J_{\perp}=5 \mathrm{~K}\right)$ are taken into account (theory II) are shown. In both cases $p=5 \times 10^{-4}, \Gamma=4 \times 10^{-4}$, and $h_{\text {res }}^{0}=7.598 \mathrm{~T}$. The material-dependent parameters are given in the text.

To compare with experiment, we assume again a Poisson distribution of chain breaks so that the measured Knight shift is given by an average over all possible chain lengths. Furthermore, each site in a chain of length $N$ gives a different Knight shift according to (5.1). If we assume that each of these Knight shifts has a Lorentzian lineshape with width $\Gamma$ we find for the NMR spectrum

$$
P(K)=\frac{\Gamma}{\pi} \sum_{N=1}^{\infty} \frac{p(1-p)^{N-1}}{N} \sum_{j=1}^{N} \frac{1}{\left(K-K_{j}^{(N)}\right)^{2}+\Gamma^{2}}
$$

Using the results for the uniform (3.18) and the staggered part (3.21) we can now immediately calculate the NMR spectrum for an ensemble of isotropic Heisenberg chain segments and compare to experimental data for $\mathrm{Sr}_{2} \mathrm{CuO}_{3}$ obtained by Takigawa et al. [6,7]. We use $J=2200 \mathrm{~K}$ as exchange constant in (1.1) and hyperfine coupling constants $A_{c}^{0} /\left(2 \hbar \gamma_{n}\right) \approx-13 \mathrm{~T}, A_{a b}^{0} /\left(2 \hbar \gamma_{n}\right) \approx 2 \mathrm{~T}$, and $A^{1} /\left(2 \hbar \gamma_{n}\right) \approx 4 \mathrm{~T}[39]$. Here the index denotes the magnetic field direction. We calculate the spectrum as a function of $h=(1+K) h_{\mathrm{res}}^{0}$ and use $h_{\mathrm{res}}^{0}=7.598 \mathrm{~T}$. For the resonance field $\nu=86 \mathrm{MHz}$ used in the experiment [6] this is consistent with $h_{\text {res }}^{0}=\nu / \gamma_{n}$ where $\gamma_{n} \approx 11.3 \mathrm{MHz} / \mathrm{T}$ [40]. A comparison of formula (5.2) for this set of material-dependent parameters with experiment is shown in figure 7 . Here the impurity concentration $p$ and the Lorentzian linewidth $\Gamma$ have been used as fitting parameters. Note that the sample used for the NMR experiment has been annealed, thus dramatically reducing the amount of excess oxygen and the associated chain breaks compared to the sample used for the susceptibility measurements shown in figure 6 . The theory predicts a central peak corresponding to the bulk susceptibility value and two shoulders with separation $\Delta h$ (see curve 'theory I' in figure 7) which are caused by the maxima of the local susceptibility (see, for example, figure 2). Theoretically, we find that $\Delta h \sim h_{\text {res }}^{0} \sqrt{v / T} \ln ^{1 / 4}(v / T)$, i. e., the separation of the shoulders increases $\sim 1 / \sqrt{T}$ with decreasing temperature. This is in agreement 
with experimental findings [6]. The central peak, however, has a much more complex structure than predicted. The peak is split (feature $\delta h$ in figure 7 ) and has prominent shoulders (feature $D h$ in figure 7 ). These features are only observed in experiment at temperatures $T \lesssim 60 \mathrm{~K}$, whereas at higher temperatures the theoretical prediction agrees well with experimental data (not shown).

To explain the additional features in the NMR spectra at $T \lesssim 60 \mathrm{~K}$ we have to take the interchain coupling into account. $\mathrm{Sr}_{2} \mathrm{CuO}_{3}$ shows three-dimensional Néel ordering at $T_{N} \sim 5 \mathrm{~K}$. According to the usual chain mean field argument the interchain coupling should, therefore, also be of the order of $J_{\perp} \sim 5 \mathrm{~K}$. This estimate is consistent with band structure calculations [8]. As we have already demonstrated numerically in figure 4, a ladder-like interchain coupling as present in $\mathrm{Sr}_{2} \mathrm{CuO}_{3}$ leads to a reflection of susceptibility oscillations. Therefore, another typical Knight shift is expected to be present in the NMR spectra, related to the maxima of the reflected oscillations. As long as $J_{\perp} / T \ll 1$ we can calculate this effect perturbatively. Starting from the definition of the local susceptibility given in equation (1.2) we obtain to the first order

$$
\chi_{j, 1}^{(1)}=-\frac{J_{\perp}}{T^{2}} \sum_{k} \underbrace{\left\langle S_{j, 1}^{z} S_{k, 1}^{z}\right\rangle}_{G_{1}^{z z}(j-k)} \underbrace{\left\langle S_{t o t, 2}^{z} S_{k, 2}^{z}\right\rangle}_{\chi_{k, 2}} .
$$

Here, the lower index 1 stands for the infinitely long chain without chain breaks whereas the lower index 2 denotes the chain with a chain break at site $k=0$. Both, the two-point correlation $G_{1}^{z z}(j-k)$ and the local susceptibility $\chi_{k, 2}$ have a uniform and a staggered part in the low temperature limit.

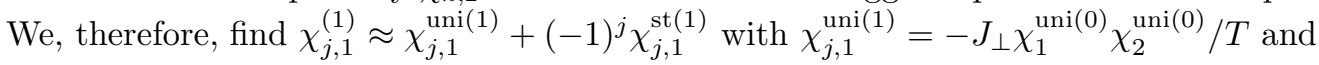

$$
\chi_{j, 1}^{\mathrm{st}(1)}=-\frac{J_{\perp}}{T}(-1)^{j} \sum_{k} \chi_{k, 2}^{\mathrm{st}} G_{1}^{z z, s t}(j-k) .
$$

Here equation (3.8) has to be used for $\chi_{k, 2}^{\mathrm{st}}$ while $G_{1}^{z z, s t}(j-k)=\left\langle S_{j}^{z} S_{k}^{z}\right\rangle^{\mathrm{st}}=c^{2} /\left[\frac{v}{\pi T} \sinh \left(\frac{\pi T}{v}|j-k|\right)\right]^{K}$ is the staggered part of the bulk two-point correlation function. In figure 4 , formula (5.4) is compared with the numerical result and good agreement is found.

If we take these reflections into account, then also the additional features in the NMR spectra are explained as shown in figure 7 (curve 'theory II'). In particular, the shoulders with separation $D h$ directly correspond to the maxima of the reflected susceptibility oscillations. The splitting of the peak $\delta h$ is of different origin. It is in fact not a splitting but rather a loss of intensity at the value which corresponds to the bulk susceptibility. The oscillations and reflected oscillations spread over the entire crystal at low temperatures so that there are simply no sites left which show bulk behavior. Rather interestingly, the peak usually associated with the bulk susceptibility value, therefore, turns into a dip at low temperatures due to the presence of chain breaks and interchain couplings.

Finally, we also want to shed some light on the role played by the geometry of the interchain couplings. To this end, we consider an interchain coupling as shown in figure 1c. Such a coupling is realized in $\mathrm{SrCuO}_{2}$ with a ferromagnetic $J_{\perp} \sim[-0.1 J,-0.3 J][1,8]$. Using again first order perturbation theory we find in this case

$$
\chi_{j, 1}^{(1)}=-\frac{J_{\perp}}{T^{2}} \sum_{k} \underbrace{\left\langle S_{j, 1}^{z} S_{k, 1}^{z}\right\rangle}_{G_{1}^{z z}(j-k)} \underbrace{\left\langle S_{t o t, 2}^{z}\left(S_{k-1,2}^{z}+S_{k, 2}^{z}\right)\right\rangle}_{\chi_{k-1,2}+\chi_{k, 2}} .
$$

Separating this into a uniform and a staggered part we find $\chi_{j, 1}^{\mathrm{uni}(1)}=-2 J_{\perp} \chi_{1}^{\mathrm{uni}(0)} \chi_{2}^{\mathrm{uni}(0)} / T$ and

$$
\chi_{j, 1}^{\mathrm{st}(1)}=-\frac{J_{\perp}}{T}(-1)^{j} \sum_{k} \underbrace{\left(\chi_{k, 2}^{\mathrm{st}}-\chi_{k-1,2}^{\mathrm{st}}\right)}_{\approx 0} G_{1}^{z z, s t}(j-k) .
$$

Therefore, no reflections will occur in this case to first order in perturbation theory consistent with the numerical results shown in figure 5. This also means that an NMR spectrum for $\mathrm{SrCuO}_{2}$ would not show any shoulders associated with the coupling to the nearest neighbor chain. Reflections in chains further away might, however, be still possible which then would again lead to additional structures in the NMR spectra at low temperatures. 


\section{Summary and conclusions}

We have investigated here how chain breaks and interchain couplings affect the physical properties of spin chain compounds. A weak coupling of two Heisenberg chains is an irrelevant perturbation in the renormalization group sense whereas the weakening of a bond in an otherwise homogenous chain is relevant. Open boundary conditions are, therefore, the stable fix point. Due to this reasoning it is expected that a wide class of perturbations like impurities or dislocations present in any real compound can be effectively described at low energies as a chain break. An experimental measurement then corresponds to taking an average over an ensemble of finite chain segments with open boundaries.

By combining a low-energy effective field theory with Bethe ansatz we have derived parameterfree formulas for the thermodynamics of finite spin-1/2 Heisenberg chains with open boundary conditions. Particular emphasis was put on a calculation of the susceptibility. Due to the broken translational invariance there exists a site-dependent staggered susceptibility in addition to the uniform site-independent part. However, even the uniform part is affected by the open boundary conditions in the sense that a surface contribution arises which is not present for periodic boundary conditions.

We have shown that susceptibility measurements on $\mathrm{Sr}_{2} \mathrm{CuO}_{3}$ doped with non-magnetic $\mathrm{Pd}$ ions are well described by the theory presented here. One of the complications arising for this compound is, however, that even the undoped sample apparently already has a relatively large amount of chain breaks which are believed to be caused by excess oxygen. The impurity concentration used in the theory to fit the experimental data, therefore, differs significantly from the nominal Pd concentration. The emerging picture nevertheless seems to be consistent - at least at low impurity concentrations - with a fixed concentration of additional chain breaks already present in the undoped sample. It would certainly be of some value to obtain experimental data for this or some other spin-chain compound where the impurity concentration is well controlled and a direct comparison with theory is, therefore, possible.

Furthermore, we used the field theory to calculate NMR spectra. Importantly, the Knight shift is proportional to the local susceptibility so that the staggered site-dependent part of the susceptibility, which cannot be observed in a bulk susceptibility measurement, becomes observable. As has already been shown previously [6], the staggered susceptibility leads to a broad background in the NMR spectra with edges caused by the maxima of the staggered susceptibility. For $\mathrm{Sr}_{2} \mathrm{CuO}_{3}$ it has, however, been found that the NMR spectra at low temperatures show puzzling additional features which have been ascribed in [7] to a coupling to phonons. Here we have shown that these features quite naturally arise in a spin-only model if the known interchain couplings are taken into account. It is also important to note that the geometry of the interchain couplings is crucial. As a specific example we also considered, in addition to the ladder-like interchain coupling relevant for $\mathrm{Sr}_{2} \mathrm{CuO}_{3}$, the zigzag-like interchain coupling realized in $\mathrm{SrCuO}_{2}$. In the latter case no additional structures in the NMR spectra related to this interchain coupling will occur. NMR experiments on spin chain compounds are, therefore, not only helpful to study of impurity effects. They can also be used to investigate the geometry and strength of interchain couplings.

\section{Acknowledgements}

I want to thank all my collaborators on this and related topics, in particular, Ian Affleck, Michael Bortz, Sebastian Eggert, Andreas Klümper, and Nicolas Laflorencie.

\section{References}

1. Motoyama N., Eisaki H., Uchida S., Phys. Rev. Lett., 1996, 76, 3212.

2. Pratt F.L., Blundell S.J., Lancaster T., Baines C., Takagi S., Phys. Rev. Lett., 2006, 96, 247203.

3. Thurber K.R., Hunt A.W., Imai T., Chou F.C., Phys. Rev. Lett., 2001, 87, 247202.

4. Sologubenko A.V., Giannó K., Ott H.R., Vietkine A., Revcolevschi A., Phys. Rev. B, 2001, 64, 054412.

5. Kojima K. et al., Phys. Rev. B, 2004, 70, 094402.

6. Takigawa M., Motoyama N., Eisaki H., Uchida S., Phys. Rev. B, 1997, 55, 14129.

7. Boucher J.P., Takigawa M., Phys. Rev. B, 2000, 62, 367. 
8. Rosner H., Eschrig H., Hayn R., Drechsler S.L., Málek J., Phys. Rev. B, 1997, 56, 3402.

9. Hase M., Phys. Rev. Lett., 1993, 70, 3651.

10. Ma S., Broholm C., Reich D.H., Sternlieb B.J., Erwin R.W., Phys. Rev. Lett., 1992, 69, 3571.

11. Rüegg C. et al., Phys. Rev. Lett., 2008, 101, 247202.

12. Eggert S., Affleck I., Takahashi M., Phys. Rev. Lett., 1994, 73, 332.

13. Eggert S., Affleck I., Phys. Rev. B, 1992, 46, 10866.

14. Giamarchi T. Quantum Physics in One Dimension. Clarendon Press, Oxford, 2004.

15. Zotos X., Naef F., Prelovšek P., Phys. Rev. B, 1997, 55, 11029.

16. Sirker J., Laflorencie N., Fujimoto S., Eggert S., Affleck I., Phys. Rev. Lett., 2007, 98, 137205.

17. Sirker J., Laflorencie N., Fujimoto S., Eggert S., Affleck I., J. Stat. Mech., 2008, P02015.

18. Fujimoto S., Eggert S., Phys. Rev. Lett., 2004, 92, 037206.

19. Furusaki A., Hikihara T., Phys. Rev. B, 2004, 69, 094429.

20. Bortz M., Sirker J., J. Phys. A: Math. Gen., 2005, 38, 5957.

21. Sirker J., Bortz M., J. Stat. Mech., 2006, P01007.

22. Sirker J., Bortz M., Phys. Rev. B, 2006, 73, 1.

23. Bursill R. et al., J. Phys: Cond. Mat., 1995, 7, 8605.

24. Wang X., Xiang T., Phys. Rev. B, 1997, 56, 5061.

25. Shibata N., J. Phys. Soc. Jpn., 1997, 66, 2221.

26. Sirker J., Klümper A., Phys. Rev. B, 2002, 66, 245102.

27. Sirker J., Klümper A., Europhys. Lett., 2002, 60, 262.

28. Sirker J., Klümper A., Phys. Rev. B, 2005, 71, 241101(R).

29. Trotter H.F., Proc. Amer. Math. Soc., 1959, 10, 545.

30. Suzuki M., Commun. Math. Phys., 1976, 51, 183.

31. Suzuki M., Phys. Rev. B, 1985, 31, 2957.

32. Eggert S., Rommer S., Phys. Rev. Lett., 1998, 81, 1690.

33. Klümper A., Raupach R., Schönfeld F., Phys. Rev. B, 1999, 59, 3612.

34. Eggert S., Affleck I., Phys. Rev. Lett., 1995, 75, 934.

35. Affleck I., J. Phys. A, 1998, 31, 4573.

36. Lukyanov S., Terras V., Nucl. Phys. B, 2003, 654, 323.

37. Sirker J., Laflorencie N., EPL, 2009, 86, 57004.

38. Lukyanov S., Nucl. Phys. B, 1998, 522, 533.

39. Monien H., Pines D., Takigawa M., Phys. Rev. B, 1990, 43, 258.

40. Abragam A., Bleaney B. Electron Paramagnetic Resonance of Transition Ions. Clarendon Press, Oxford, 1970.

\title{
Наслідки недосконалостей гратки і міжланцюжкових взаємодій для критичних властивостей спін-1/2 ланцюжкових сполук
}

\author{
€. Сіркер \\ 1 Факультет фізики і дослідницький центр OPTIMAS, Університет Кайзершлаутена, Німеччина \\ 2 Інститут досліджень твердого стану Макса Планка, вул. Гайзенберга 1, D-70569 Штутгарт, \\ Німеччина
}

Отримано 7 травня 2009 р.

\begin{abstract}
Щоб мати можливість порівняти теоретичні передбачення для спінових ланцюжків з експериментальними даними, часто важливо взяти до уваги ефекти домішок, так само, як міжланцюжкові взаємодії. Тут ми представляємо теорію поля для скінчених спінових ланцюжків при скінченій температурі і обчислюємо експериментально вимірювані величини як сприйнятливості і спектри ядерного магнітного резонансу. Для міжланцюжкових взаємодій ми зосереджуємося на геометріях, істотних для купратних спінових ланцюжків як от $\mathrm{Sr}_{2} \mathrm{CuO}_{3}$ i $\mathrm{SrCuO}_{2}$. Теоретико-польові результати порівнюються з експериментальними і чисельними даними, отриманими з використанням ренормалізаційної групи для матриці густини.
\end{abstract}

Ключові слова: спінові ланцюжки, домішки, термодинаміка, бозонізація, ренормалізаційна група для матрицігустини

PACS: $75.10 . P q, 75.10 . J m, 11.10 . W x, 0230.1 k$ 